\title{
Stochastic Bifurcation in Generalized Chua's Circuit through Alpha-Stable Levy Noise
}

\author{
Ferit Acar Savacı \\ Department of Electrical and Electronics Engineering \\ Izmir Institute of Technology \\ Izmir, Turkey 35430 \\ Email: acarsavaci@iyte.edu.tr
}

\author{
Serpil Y1lmaz \\ Department of Electrical and Electronics Engineering \\ Izmir Institute of Technology \\ Izmir, Turkey 35430 \\ Email: serpilyilmaz@iyte.edu.tr
}

\begin{abstract}
In this study, we investigate the changes in the dynamics of generalized Chua's circuit through $\alpha$-stable Lévy noise in the framework of stochastic bifurcation concept. By choosing the noise intensity, characteristic exponent (impulsiveness parameter) and the skewness parameter of the $\alpha$-stable Lévy noise as the bifurcation parameters we have observed the qualitative changes in the stationary probability distributions and in the structures of the stochastic attractors.
\end{abstract}

\section{INTRODUCTION}

The effects of noise on the system dynamics due to the internal or external fluctuations are inevitable in the real world systems and therefore the stochastic resonance, the noiseinduced transitions and stochastic bifurcations have attracted a great deal of attention in the many diverse fields of the science [1]-[5]. Stochastic bifurcations have been defined as P-bifurcation and D-bifurcation in [6], [7]. Phenomenological bifurcation (P-bifurcation) is characterized by the qualitative change in the stationary probability distributions and Dynamical bifurcation (D-bifurcation) is observed by the changes in the sign of Lyapunov exponents. The changes in the attractors of Chua's circuit driven by Wiener process have been analyzed in [8]. In [4] the influence of Gaussian noise on Duffing-Van der Pol and synthetic genetic oscillators have been investigated by the concept of stochastic bifurcation. A similar system with parametric noise have been investigated in [9].

Limit cycles of the Lorenz system driven by the Wiener process have been analyzed by P-bifurcation and D- bifurcation in [10] and the effect of time delay on the stochastic bifurcation in Van der Pol oscillator perturbed by white noise have been investigated in [11]. Duffing oscillator with friction excited by Poisson white noise and its stochastic bifurcation analysis have been studied in [12]. The P-bifurcation in a birhythmic Van der Pol like oscillator subject to exponentially correlated noises and in a bistable fractional-order system have been investigated in [13], [14], respectively.

Since the random fluctuations in many real physical phenomena show impulsive and asymmetric characteristics then such an impulsive and asymmetric random fluctuations with heavy-tailed and skewed distributions are modeled by stable non-Gaussian distributions (referred to as alpha-stable ( $\alpha$ stable) distributions) [15], [16]. Stochastic $\alpha$-stable Lévy type dynamical system is more general class of random dynamical systems [17], [18] and its application areas are very wide including the power systems, neural networks, finance, social networks, biological systems [19]-[25]. Bifurcations for a simple nonlinear dynamical system under additive Lévy noises have been analyzed in [26].

In this paper generalized Chua's circuit driven by $\alpha$-stable Lévy noise have been analyzed by solving its corresponding Itô stochastic differential equation (SDE) numerically using Euler-Maruyama method. The variations in the structure of the attractors and the stationary distributions of the responses have been observed by changing the characteristic exponent (impulsiveness parameter), skewness and the intensity parameters of $\alpha$-stable Lévy noise while fixing the number of breakpoints in piecewise-linear characteristics of Generalized Chua's Circuit (GCC). As a future work we are planning to construct new random communication scheme based on stochastic shift keying [27], [28] in which by varying the paramters of $\alpha$-stable noise the changes in the stochastic attractors are used to decode the binary message signal.

\section{Generalized Chua's Circuit Driven By AlPHA-STABle LEVy NOISE}

The dynamics of stochastic dimensionless GCC can be described by the following set of three differential equations

$$
\begin{aligned}
d x & =a[y-h(x)] d t \\
d y & =(x-y+z) d t \\
d z & =-b y d t+\epsilon \xi(t) d t
\end{aligned}
$$

where $a$ and $b$ denote bifurcation parameters, and the piecewise-linear characteristics $h(x)$ is given as

$$
h(x)=m_{2 q-1} x+\frac{1}{2} \sum_{i=1}^{2 q-1}\left(m_{i-1}-m_{i}\right)\left(\left|x+c_{i}\right|-\left|x-c_{i}\right|\right)
$$

The number of breakpoints " $q "$ determines the number of scrolls.

$\xi(t)$ indicates alpha-stable Lévy distributed noise and $\epsilon$ is the noise intensity then by defining the state variable as $\mathbf{X}=$ $\left[\begin{array}{lll}x & y & z\end{array}\right]^{T}$, (1) can be rewritten in the Itô form of SDE :

$$
d \mathbf{X}=\mathbf{f}(\mathbf{X}) d t+\mathbf{g} d L_{\alpha}(t)
$$


where

$$
\mathbf{f}=\left[\begin{array}{c}
\alpha(y-h(x)) \\
x-y+z \\
-\beta y
\end{array}\right] ; \mathbf{g}=\left[\begin{array}{l}
0 \\
0 \\
\epsilon
\end{array}\right]
$$

and the increments of the Lévy process $d L_{\alpha}(t)$ is $\alpha$-stable random variable [15].

\section{A. Alpha-stable random variable}

There is no closed-form expression for the probability density function of $\alpha$-stable distributions, however it can be calculated by the inverse Fourier transform of the characteristic function given as

$$
\phi(t)=\left\{\begin{array}{cc}
\exp \left\{j \mu t-\gamma^{\alpha}|t|^{\alpha}\left(1-j \beta \operatorname{sign}(t) \tan \left(\frac{\alpha \pi}{2}\right)\right)\right\} & \text { if } \alpha \neq 1 \\
\exp \left\{j \mu t-\gamma|t|\left(1+j \beta \frac{2}{\pi} \operatorname{sign}(t) \ln \left(\frac{\alpha \pi}{2}\right)\right)\right\} & \text { if } \alpha=1
\end{array}\right.
$$

The distribution of $\alpha$-stable random variable is denoted by $\alpha$ stable distribution $S_{\alpha}(\gamma, \beta, \mu)$ which is characterized by the four parameters: $\mu$ denotes the location, $\gamma$ is scale parameter, the characteristic exponent $\alpha(0<\alpha \leq 2)$ measures the impulsiveness, and the skewness parameter $\beta$ measures the symmetry of the distribution in which $\beta=0$ refers to symmetric distribution, $\beta<0$ to left-skewed distribution and $\beta>0$ to right-skewed distribution in [15], [18], [29]. As the value of characteristic exponent " $\alpha$ " decreases then the impulsiveness increases and hence the tails of the corresponding distributions become heavier. Also, the increase in the absolute value of the $\beta$ results in the more asymmetric (skewed) distribution.

$L_{\alpha}(t): t \geq 0$ stands for $\alpha$-stable Lévy motion. It has the following properties [16], [18],

- $L_{\alpha}(0)=0$ almost surely (a.s.),

- $L_{\alpha}(\mathrm{t})$ has the independent and stationary increments $" d L_{\alpha}(t) "$

- $d L_{\alpha}(t) \doteq L_{\alpha}(t)-L_{\alpha}(s) \sim S_{\alpha}\left((t-s)^{1 / \alpha}, \beta, 0\right)$ for any $0 \leq s<t<\infty$.

Remark : The Lévy processes include random motions whose sample paths are right-continuous with left limits (cádlág function) and have countable random jump discontinuties occurring at random times on a finite time interval. Therefore, the Lévy processes include Brownian processes and the Poisson processes (to represent the large and small jumps) [18]. In fact, Brownian motion is the special case of $\alpha$-stable Lèvy motion with $\alpha=2$ and $S_{2}(\gamma, 0, \mu)=N\left(\mu, 2 \gamma^{2}\right)$ Normal (Gaussian) distribution with mean $\mu$ and variance $2 \gamma^{2}$ [15].

Typical trajectories for $\alpha$-stable Lévy motion are shown in Fig. 1 which have been used as stochastic excitations of GCC in (1).

The approximate numerical solution of Eq. (3) can be obtained by using the Euler-Maruyama method given in [16], [30] as

$$
\mathbf{X}_{t_{i}}=\mathbf{X}_{t_{i-1}}+\mathbf{f}\left(\mathbf{X}\left(t_{i-1}\right)\right) \tau+\mathbf{g} \triangle L_{\alpha, i}^{\tau}
$$

where the increment of the Lévy process is $\alpha$-stable random variable $\triangle L_{\alpha, i}^{\tau}$ defined by

$$
\triangle L_{\alpha, i}^{\tau}=L_{\alpha}\left(\left[t_{i-1}, t_{i}\right]\right) \sim S_{\alpha}\left(\tau^{1 / \alpha}, \beta, \mu\right)
$$

with $\tau=t_{i}-t_{i-1}$ have been generated by the method given in [15].

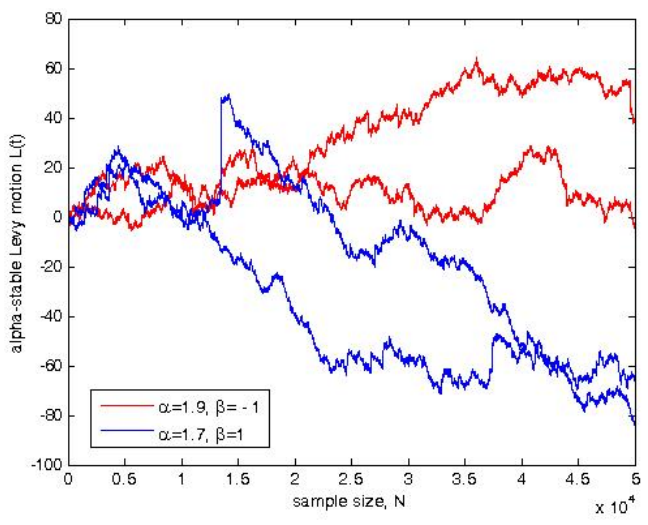

Fig. 1. $\alpha$-stable Lévy motion.

\section{P-BIfuRCATION OF STOCHASTIC GCC}

The GCC with 5-scroll attractor has been chosen as the case study in which parameters have been chosen as $a=9$, $b=14.286, q=4$. The parameters of the piecewise linear characteristics of (2) are fixed as $c_{1}=1, c_{2}=2.15, c_{3}=$ $3.6, c_{4}=6.2, c_{5}=9, m_{0}=0.9 / 7, m_{1}=-3 / 7, m_{2}=$ $3.5 / 7, m_{3}=-2.7 / 7, m_{4}=4 / 7, m_{5}=-2.4 / 7$.

The dynamical behaviors of the system are investigated numerically by varying the characteristic exponent $\alpha$, the skewness parameter $\beta$ and the noise intensity $\epsilon$. In all of the figures the phase plots and the corresponding histograms $p(x)$ and $p(y)$ of the states $x-y$ are obtained from the 100 realizations with the time step $\tau=0.01$.

Fig. 2 shows that in the presence of Brownian motion with the noise intensity $\epsilon=0.008$ there is no change in the structure of the attractor of GCC, it preserves the structure of 5-scrolls as in the deterministic case. However with the impulsive and asymmetric $\alpha$-stable Lévy motion, the structure of the multiscroll attractors of GCC has been changed as shown Fig. 3Fig. 8.

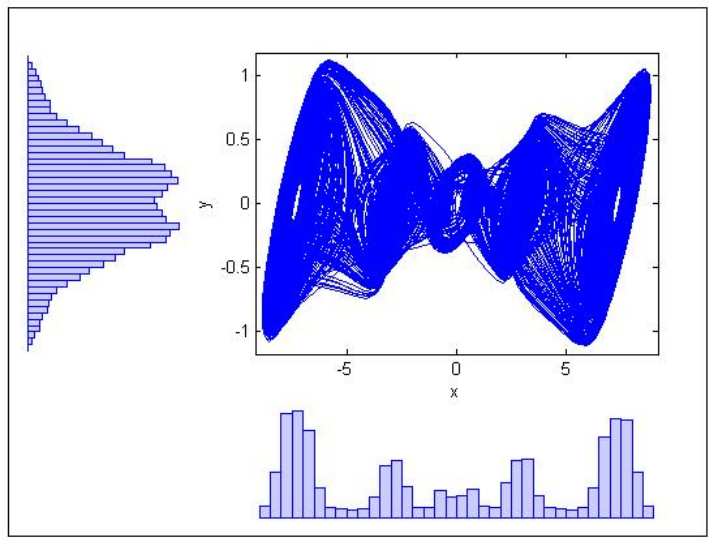

Fig. 2. Brownian motion $(\alpha=2.0, \beta=0)$ with $\epsilon=0.008$. 


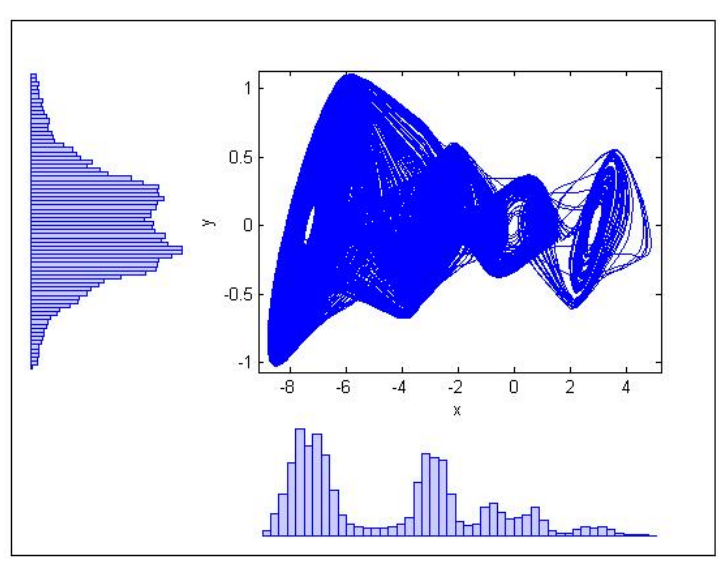

Fig. 3. $\alpha$-stable Lévy motion with $\alpha=1.7, \beta=1$ and $\epsilon=0.008$.

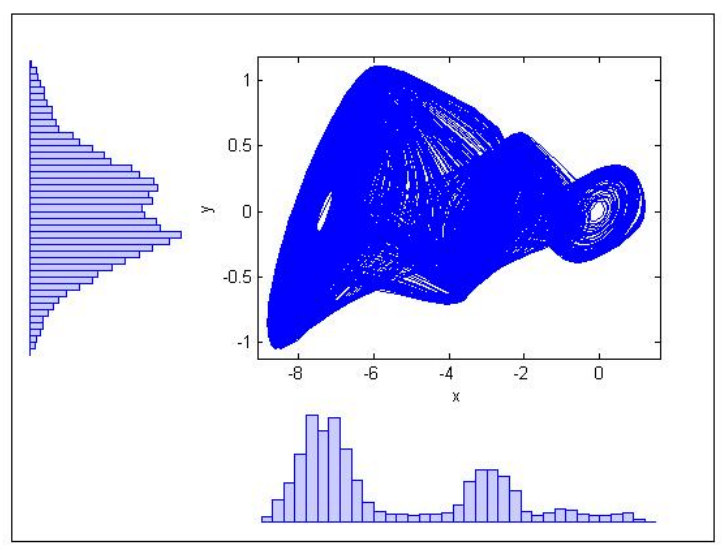

Fig. 4. $\alpha$-stable Lévy motion with $\alpha=1.6, \beta=1$ and $\epsilon=0.001$.

For $\alpha$-stable Lévy motion with $\alpha=1.7, \beta=1$ and $\epsilon=0.008$ more than 90 percent of 100 realizations exhibit 4-scroll attractor as shown in Fig. 3. For Lévy motion with $\alpha=1.6, \beta=1$ and $\epsilon=0.001$ more than 90 percent of 100 realizations exhibit 3 -scroll attractor while Lévy motion with $\alpha=1.2, \beta=1$ and $\epsilon=0.001$ generates 2-scrolls attractor as shown in Fig. 4 and Fig. 5, respectively.

For Lévy motion with $\beta=-1$ by varying the characteristic exponent $\alpha$ and the noise intensity $\epsilon$ the responses as shown in Fig. 6-Fig. 8 are observed. The results show that one of the maxima in the histogram $p(x)$ disappears and qualitative changes in the stationary histograms occur indicating the $\mathrm{P}$ bifurcation which depends on the $\alpha, \beta$ and $\epsilon$ parameters.

An increase in the impulsiveness (decrease of $\alpha$ ) results in a decrease in the number of scrolls. It can be seen from Fig. 4 - Fig. 7 that when the norm of skewness parameter and noise intensity are not changed, as the characteristic exponent $\alpha$ becomes smaller the number of scrolls becomes lesser.

The decrease in the number of scrolls can be achieved even if applying low noise intensity while the characteristic exponent of the noise is chosen smaller (the impulsiveness is increased).

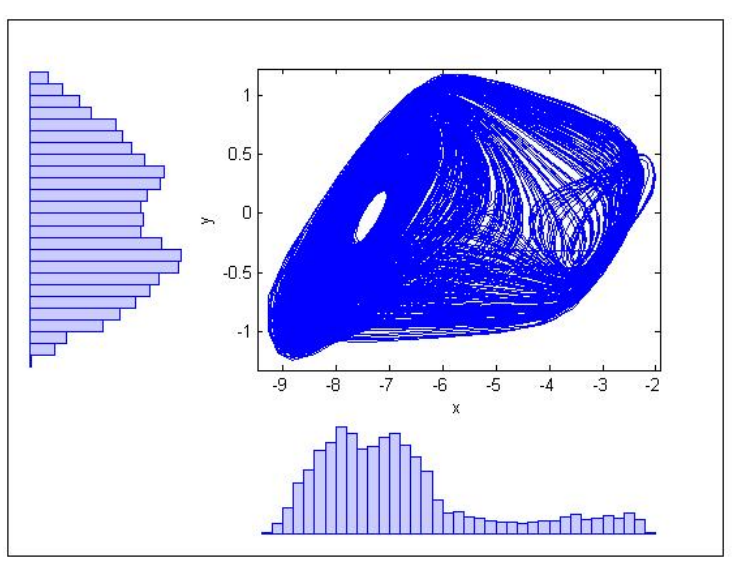

Fig. 5. $\alpha$-stable Lévy motion with $\alpha=1.2, \beta=1$ and $\epsilon=0.001$.

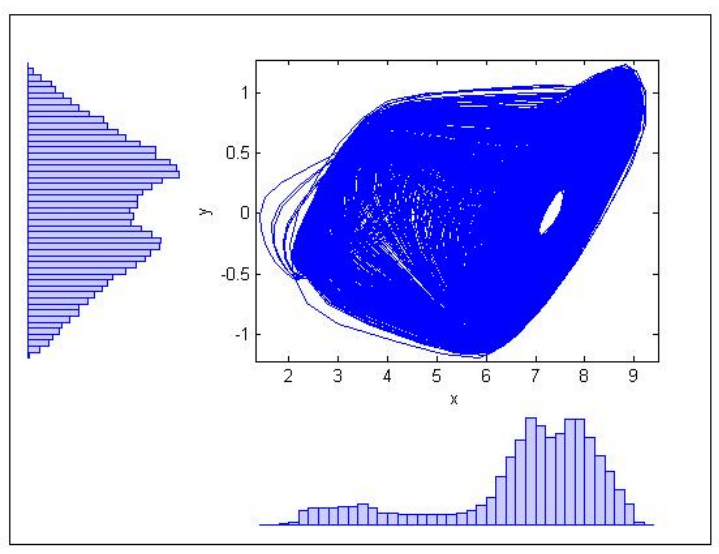

Fig. 6. $\alpha$-stable Levy motion with $\alpha=1.2, \beta=-1$ and $\epsilon=0.001$.

The other important observation is that the locations of scroll of attractors depend on the sign of the skewness parameters $\beta$.

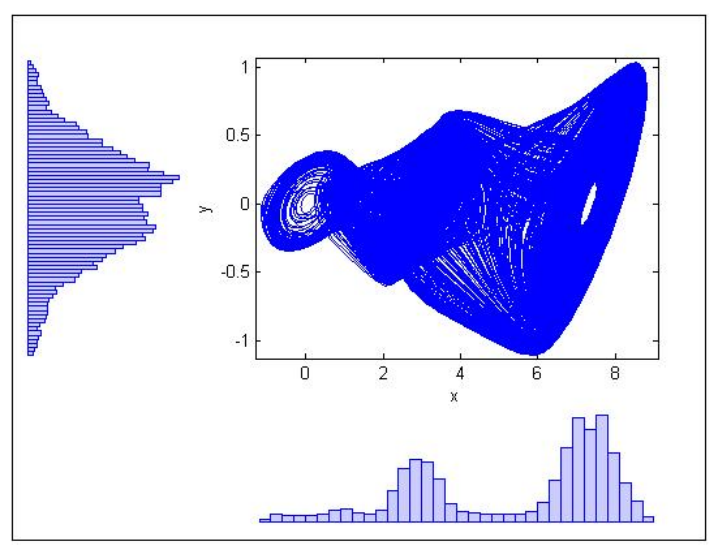

Fig. 7. $\alpha$-stable Levy motion with $\alpha=1.6, \beta=-1$ and $\epsilon=0.001$. 


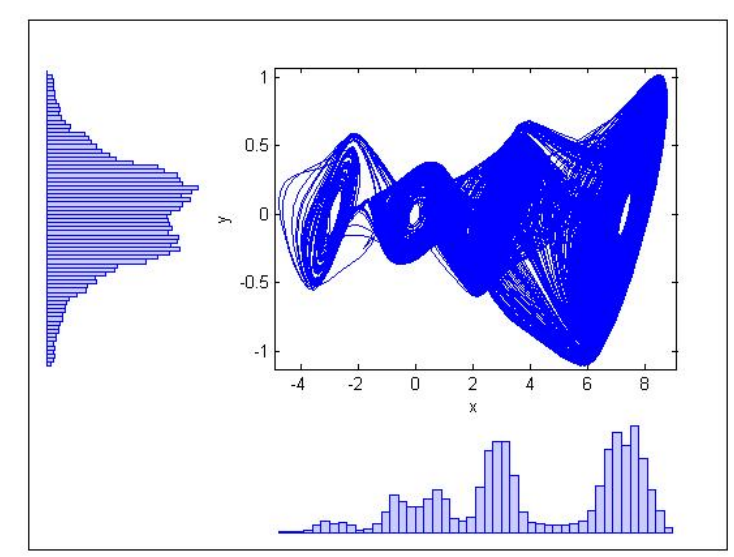

Fig. 8. $\alpha$-stable Levy motion with $\alpha=1.9, \beta=-1$ and $\epsilon=0.01$.

\section{CONCLUSION}

In this study, the deterministic generalized Chua's circuit with 5-scroll attractor has been specifially chosen and by applying $\alpha$-stable noise while fixing the breakpoints of the piecewise-linear characteristics of the nonlinear resistor in the GCC, the structure of the attractors of the GCC have been changed. Stochastic P-bifurcations induced by the noise parameters are observed via the qualilitative changes in the normalized histograms. Namely, the number of scrolls are decreased and transitions from multi-modal stationary distributions to bimodal distribution are observed by properly choosing the impulsiveness parameter of $\alpha$-stable Lévy noise. The results simulations show that an increase in the impulsiveness (decrease in the characteristic exponent) leads to a decrease in the number of scrolls. The skewness parameter of $\alpha$-stable Lévy noise also have clear effect on the structure of the attractors.

\section{REFERENCES}

[1] R. Benzi, A. Sutera, and A. Vulpiani, "The mechanism of stochastic resonance," Journal of Physics A: mathematical and general, vol. 14, no. 11, p. L453, 1981.

[2] L. Gammaitoni, P. Hänggi, P. Jung, and F. Marchesoni, "Stochastic resonance," Reviews of modern physics, vol. 70, no. 1, p. 223, 1998.

[3] B. Andò and S. Graziani, Stochastic resonance: theory and applications. Springer Science \& Business Media, 2012.

[4] A. Zakharova, T. Vadivasova, V. Anishchenko, A. Koseska, and J. Kurths, "Stochastic bifurcations and coherence like resonance in a self-sustained bistable noisy oscillator," Physical Review E, vol. 81, no. 1, p. $011106,2010$.

[5] V. V. Uchaikin and V. M. Zolotarev, Chance and stability: stable distributions and their applications. Walter de Gruyter, 1999.

[6] L. Arnold, "Recent progress in stochastic bifurcation theory," in $I U$ TAM Symposium on Nonlinearity and Stochastic Structural Dynamics. Springer, 2001, pp. 15-27.

[7] - "Random dynamical systems," 2013.

[8] M. Yu. Ryagin and L. B. Ryashko, "The analysis of the stochastically forced periodic attractors for Chua's circuit," International Journal of Bifurcation and Chaos, vol. 14, no. 11, pp. 3981-3987, 2004.

[9] I. Bashkirtseva, T. Ryazanova, and L. Ryashko, "Stochastic bifurcations caused by multiplicative noise in systems with hard excitement of autooscillations," Physical Review E, vol. 92, no. 4, p. 042908, 2015.

[10] I. Bashkirtseva, L. Ryashko, and P. Stikhin, "Noise-induced chaos and backward stochastic bifurcations in the lorenz model," International Journal of Bifurcation and Chaos, vol. 23, no. 05, p. 1350092, 2013.
[11] Z. Sun, J. Fu, Y. Xiao, and W. Xu, "Delay-induced stochastic bifurcations in a bistable system under white noise," Chaos: An Interdisciplinary Journal of Nonlinear Science, vol. 25, no. 8, p. 083102, 2015.

[12] P. Kumar, S. Narayanan, and S. Gupta, "Stochastic bifurcation analysis of a duffing oscillator with coulomb friction excited by poisson white noise," Procedia Engineering, vol. 144, pp. 998-1006, 2016.

[13] R. M. Yonkeu, R. Yamapi, G. Filatrella, and C. Tchawoua, "Stochastic bifurcations induced by correlated noise in a birhythmic van der pol system," Communications in Nonlinear Science and Numerical Simulation, vol. 33, pp. 70-84, 2016.

[14] J. Yang, M. A. Sanjuán, H. Liu, G. Litak, and X. Li, "Stochastic p-bifurcation and stochastic resonance in a noisy bistable fractionalorder system," Communications in Nonlinear Science and Numerical Simulation, vol. 41, pp. 104-117, 2016.

[15] G. Samorodnitsky and M. S. Taqqu, Stable non-Gaussian random processes: stochastic models with infinite variance. CRC press, 1994, vol. 1.

[16] A. Janicki and A. Weron, Simulation and chaotic behavior of alphastable stochastic processes. CRC Press, 1993, vol. 178.

[17] M. Grigoriu, "Linear systems subject to non-gaussian $\alpha$-stable processes," Probabilistic engineering mechanics, vol. 10, no. 1, pp. 23-34, 1995.

[18] D. Applebaum, Lévy processes and stochastic calculus. Cambridge university press, 2009.

[19] S. Yilmaz and F. A. Savacı, "Effect of levy type load fluctuations on the stability of single machine infinite bus power systems," in Circuit Theory and Design (ECCTD), 2017 European Conference on. IEEE, 2017.

[20] A. Patel and B. Kosko, "Stochastic resonance in continuous and spiking neuron models with levy noise," IEEE Transactions on Neural Networks, vol. 19, no. 12, pp. 1993-2008, 2008.

[21] W. Zhou, J. Yang, L. Zhou, and D. Tong, Stability and Synchronization Control of Stochastic Neural Networks. Springer, 2015, vol. 35.

[22] M. Ibragimov, R. Ibragimov, and J. Walden, Heavy-tailed distributions and robustness in economics and finance. Springer, 2015, vol. 214.

[23] P. Embrechts, C. Klüppelberg, and T. Mikosch, Modelling extremal events: for insurance and finance. Springer Science \& Business Media, 2013, vol. 33.

[24] Z. Cui and Y. Xu, "Social emotional optimisation algorithm with levy distribution," International Journal of Wireless and Mobile Computing, vol. 5, no. 4, pp. 394-400, 2012.

[25] Y. Xu, J. Feng, J. Li, and H. Zhang, "Stochastic bifurcation for a tumorimmune system with symmetric lévy noise," Physica A: Statistical Mechanics and its Applications, vol. 392, no. 20, pp. 4739-4748, 2013.

[26] C. Huiqin, D. Jinqiao, and Z. Chengjian, "Elementary bifurcations for a simple dynamical system under non-gaussian lévy noises," Acta Mathematica Scientia, vol. 32, no. 4, pp. 1391-1398, 2012.

[27] M. E. Çek and F. Savaci, "Stable non-gaussian noise parameter modulation in digital communication," Electronics Letters, vol. 45, no. 24, pp. 1256-1257, 2009.

[28] A. Ahmed and F. A. Savaci, "Random communication system based on skewed alpha-stable levy noise shift keying," Fluctuation and Noise Letters, p. 1750024, 2017.

[29] C. L. Nikias and M. Shao, Signal processing with alpha-stable distributions and applications. Wiley-Interscience, 1995.

[30] P. E. Kloeden, E. Platen, and H. Schurz, "Numerical solution of sde through computer experiments," 1994. 